WIENER SLAVISTISCHES JAHRBUCH, Band 55/2009, 253-260

(C) 2009 by Österreichische Akademie der Wissenschaften, Wien

S A ŠK A S̆ T U M B ER G E R

\title{
Slowenische Sprache und Zweisprachigkeit bei den Slowenen in Deutschland
}

Der Beitrag stellt eine Untersuchung zur slowenischen Sprache und Zweisprachigkeit der Slowenen in Deutschland vor, die ich von 1999 bis 2004 während meiner Arbeit als Slowenisch-Lektorin am Slavischen Seminar der Universität in Tübingen durchgeführt habe. Im empirischen Teil wird die Lage der Slowenen in Deutschland, ihre Zweisprachigkeit und ihr Verhältnis zum Deutschen und Slowenischen beschrieben. Das Material für die Untersuchung wurde mit folgenden Methoden gesammelt: teilnehmende Beobachtung, Interview und Umfrage.

\section{EINLEITUNG}

Wenn Gruppen mit unterschiedlichen Sprachen aufeinander treffen, ist es für die Verständigung notwendig, dass eine Gruppe die Sprache der anderen lernt. Die Entscheidung für den Wechsel oder das Beibehalten einer Sprache ist mit der Lage der Sprecher verbunden. Der Sprecher, der bei seiner Sprache bleibt, zeigt damit seine stärkere Position in der Gesellschaft, da gewöhnlich die stärkere Gruppe der schwächeren die Sprache aufzwingt. Der schwächere Sprecher hat keine Wahl. Aus wirtschaftlicher Abhängigkeit und politischer Unterlegenheit heraus ist er gezwungen, mit dem Stärkeren zu kooperieren, deshalb lernt er dessen Sprache und wird zweisprachig.

Der Zusammenhang zwischen Sprachwahl und wirtschaftlicher oder politischer Position hat dazu geführt, dass Zweisprachigkeit in der Vergangenheit vor allem ein Merkmal der unteren Schichten war. „Sie galt als etwas Negatives, als etwas Unvollendetes im Prozess des Übergangs von der Einsprachigkeit in einer wenig geschätzten Minderheitensprache mit niedrigem Status über die Zweisprachigkeit in der Muttersprache mit niedrigem Status und in der Sprache der Mehrheit mit hohem Status in die Einsprachigkeit in der Sprache der Mehrheit. So gesehen wurde die 
Zweisprachigkeit als notwendiges Übel, als Mittel, mit dessen Hilfe ein Angehöriger einer Minderheit an der Macht und am Glanz der Kultur und Sprache der Mehrheit teilhaben kann, betrachtet" ${ }^{\text {“1 }}$ (Skutnabb-Kangas 1991: 84).

\section{Untersuchung der Slowenen in Deutschland}

Die Untersuchung erstreckte sich über fünf Jahre (1999-2004). In dieser Zeit habe ich mit Slowenen in Deutschland gearbeitet und an Veranstaltungen teilgenommen, die von den slowenischen Vereinen und katholischen Missionen in Deutschland organisiert wurden. Ich war also ein Teil der Untersuchungsgruppe, und aus diesem Grund haben mir die Informanten mehr vertraut als etwa einem Forscher aus Slowenien. ${ }^{2}$ Günstig für die Untersuchung war auch, dass ich sowohl das Leben in Slowenien als auch in Deutschland gekannt habe. Ich habe nämlich festgestellt, dass Untersuchungen über Migranten oft einseitig sind; sie werden entweder in den Einwanderer- oder in den Auswandererländern gemacht, dabei bleibt aber unberücksichtigt, dass Migranten in beiden Ländern leben. Die Beschreibung der Sprachen bleibt dadurch unvollständig, denn die slowenische Sprache ist für Migranten nur ein Teil ihres Sprachwissens, und Deutschland ist nur ein Teil des Umfelds, in dem Slowenisch gesprochen wird. Viele Slowenen besuchen regelmäßig Slowenien, und die zweite Generation hat Slowenisch oft während ihrer Besuche bei Verwandten gelernt. $^{3}$

1 „Smatrana je za nešto negativno, za nešto polovično u procesu prelaska sa jednojezičnosti u malo cenjenom manjinskom jeziku niskog statusa, putem dvojezičnosti u maternjem jeziku niskog statusa i jezika većine visokog statusa, do konačne jednojezičnosti na jeziku većine. Tako gledano, dvojezičnost se shvatala kao nužno zlo, kao sredstvo pomoću kojega pripadnik manjine na neki način može da učestvuje u moći i sjaju kulture i jezika većine".

${ }^{2}$ Viele Slowenen sind aus politischen Gründen nach Deutschland gekommen, weil sie in der Vergangenheit Probleme mit den jugoslawischen Behörden gehabt hatten. Das Misstrauen den Behörden gegenüber (und allen, die mit dem Staat verbunden waren, d. h. auch denjenigen, die als Lehrer tätig waren oder Untersuchungen durchgeführt haben) ist bei den älteren Generationen noch immer vorhanden, so dass U. Krevs (1992), die Anfang der 1990er Jahre eine Untersuchung durchführte, keine Informanten finden konnte, mit denen sie Interviews hätte führen können.

3 „Ich habe Slowenisch gelernt ... ich weiß nicht ... von den Eltern ... also ... und dann mehr, als ich ... ich war in jeden Ferien ... ich war in Slowenien, unten." (Peter, 2. Gen., geb. 1974). (Jaz sn se nauču slovensk ... ne vem ... od starša ... no ... pol pa več, kə sn ... jaz sem bil med vsakam počitanc ... sem bil v Sloveniji, dol.) „Ja, als ich klein war, mein Bruder und ich ... wir sind in die Ferien gegangen, im Sommer ... in ... ja ... mehrmals auch zu ... zu Ostern oder auch im Winter ... zu Weihnachten ... oder so. Sodass wir, sagen wir, zwei oder drei Mal im Jahr dort waren. (...) In der Zeit, als ich in Slowenien war, habe ich mich auch unterhalten, und so habe ich gelernt." (Anita, 2. Gen., geb. 1971). (Ja, ko sem bla majhna, moj brat in jaz ... smo šli med počitnicami, tko poleti ... in ... ja ... večkrat tudi za ... za vêlko noč al pa tudi pozimi ... za božič ... al tko. Tko, da smo bli, recimo, dvaal trikrat na leto tam. (...) Med tem časom, ko sem bla v Sloveniji, sem se tam pogovarjala, in tko sem se učila.) 


\subsection{INFORMANTEN}

Bei der Auswahl der Informanten wurden drei Kriterien der Zweisprachigkeit (Skutnabb-Kangas 1981) berücksichtigt: Einstellungen, Sprachkompetenz und Herkunft. Was das Kriterium der Einstellungen betrifft, konnten nur Informanten in die Untersuchung einbezogen werden, die sich selbst als zweisprachige Sprecher eingeschätzt haben und die an der Untersuchung teilnehmen wollten. Nach dem Kriterium der Sprachkompetenz wurden nur Slowenen eingeschlossen, die Slowenisch so gut konnten, dass man mit ihnen Gespräche auf Slowenisch führen konnte. Nach dem Kriterium der Herkunft wurden sowohl Angehörige der ersten als auch der zweiten Generation eingeschlossen, die Slowenisch und Deutsch parallel oder nacheinander gelernt haben.

Daten über die Funktionen der Sprachen wurden mit Hilfe von Beobachtung gesammelt. Für Slowenisch (und andere Sprachen der Migranten) ist ein „one-side bilingualism or marked and stable social distinctions, such that only one group in a contact situation is bilingual or such that only particular domains are open or appropriate to particular languages" (Fishman 1966: 433) charakteristisch. Von den Migranten wird erwartet, dass sie neben ihrer Sprache noch die Sprache der Umgebung lernen, d. h. das Deutsche. Die Funktionsgebiete der ersten Sprache werden so drastisch eingeschränkt, der Wert der Sprache wird aber noch durch soziale und rechtliche Diskriminierung reduziert, denn die Migrantensprachen haben ein viel geringeres Ansehen als z. B. Englisch oder Französisch. „Festzuhalten gilt, dass es die Einheitssprachenideologie und die Koppelung mit der gesellschaftlichen Unterdrückung einer fremdsprachigen Bevölkerungsgruppe sind, die die soziale (Diglossie) und die individuelle Zweisprachigkeit (Bilingualismus) im Bewusstsein der Mehrheitsbevölkerung negativ stigmatisiert haben“ (Stölting 1980: 9).

Neben den oben beschriebenen Kriterien der Zweisprachigkeit wurde die untersuchte Gruppe auch durch das Alter eingegrenzt. An der Untersuchung haben nur erwachsene zweisprachige Slowenen teilgenommen, die mehr als zehn Jahre in Deutschland leben, zwei Gespräche wurden in einer slowenischen Familie und in der slowenischen katholischen Mission in Ulm aufgenommen. Das Material umfasst zwölf 90-minütige Kassetten, deren Inhalt transkribiert wurde.

\section{Slowenisch in Deutschland}

Die Position und Bedeutung der slowenischen Sprache in Deutschland hat sich im Laufe der Zeit sehr verändert. Dies war damit verbunden, dass die Slowenen am Anfang nur für eine begrenzte Zeit nach Deutschland gekommen waren, die jedoch immer länger wurde, und dass die Einstellungen zur slowenischen Sprache auch von politischen und wirtschaftlichen Umständen beeinflusst wurden, so dass Untersuchungen aus den 1980er und 1990er Jahren (Slavec 1982, Krevs 1992) ein ganz anderes Bild zeigen als meine Untersuchung, die 2004 abgeschlossen wurde. 


\subsection{MöglichKeiten für den Gebrauch der Slowenischen Sprache}

Zweisprachige Sprecher können zwischen zwei Sprachen wählen. Sie entscheiden sich gewöhnlich je nach Gesprächsthema für eine von ihnen. Sie sprechen über das Leben in Deutschland leichter auf Deutsch, weil ihnen der slowenische Wortschatz für bestimmte Lebensumstände fehlt (vgl. Lexeme wie Mitfahrzentrale, Spätzle, Lehrling, Parteifreund, Hauptschule, Grundschule, Leiterin, wissenschaftlicher Assistent, Automobilelektronikentwicklung, Anwerbevertrag, also für außersprachliche Gegebenheiten, die es in Slowenien nicht gibt bzw. nicht gab, als die Informanten das Land verlassen haben).

Noch mehr als das Thema beeinflussen aber die Gesprächteilnehmer den Gebrauch der Sprache. Die Sprecher entscheiden sich für die slowenische Sprache nur, wenn alle Gesprächteilnehmer Slowenisch können. Sie halten sich auch in den slowenischen Organisationen und Familien an diesen Usus.

In der privaten Sphäre (Familie, Freunde, Bekannte) werden Dialekte gesprochen. Für Mischehen hat ein slowenischer Lehrer in Nordrhein-Westfalen, Dušan Čegovnik, festgestellt, dass ,wenn die Mutter Slowenin ist, [die Kinder - S. Š.] die slowenische Sprache noch beherrschen, wenn jedoch nicht, so lernen sie auf Initiative des slowenischen Vaters am häufigsten Slowenisch als Fremdsprache ${ }^{\text {“4 }}$ (Rodna gruda $48 / 5,45,2001)$.

In der öffentlichen Sphäre wird die Standardsprache verwendet. In Deutschland wird dabei am meisten die deutsche Sprache gesprochen, Ausnahmen sind slowenische Vereine, der Slowenischunterricht in der Schule, die Kirche (slowenische Priester und slowenische katholische Missionen) und die Lektorate an den Universitäten.

\subsubsection{SLOWENISCHE Vereine}

Die meisten slowenischen Vereine wurden in den 1970er Jahren gegründet, bis 1978 alleine elf (Drnovšek 1996: 363). Vor 1991 konnten sich Slowenen auch in den jugoslawischen Vereinen versammeln, sie haben sich aber oft kritisch über sie geäußert. Ingrid Slavec (1982) schreibt, dass es in Mannheim einen jugoslawischen Klub gab, in dem sich die Slowenen nicht wohl gefühlt haben, weil es dort nur selten slowenische Veranstaltungen gab.

In den 1990er Jahren wurden neue slowenische Vereine gegründet, denen es aber heute an Geld und Mitglieden mangelt: „Viele Klubs sind wegen finanzieller Probleme und Sektiererei heruntergekommen, weil die Demokratie unterschiedlich verstanden wird. Viel Unzufriedenheit verursachen auch Streitereien unter den Vereinen, Neid, politische Intrigen, was zum Boykottieren von Veranstaltungen und zur

4 ,[...] če je mati Slovenka, še obvladajo slovenski jezik, če pa ni, se najpogosteje učijo slovenščine na pobudo očeta slovenskega rodu v slovenski šoli kot tuji jezik“. 
Organisation attraktiverer Veranstaltungen zur selben Zeit führt ${ }^{* 5}$ (Rodna gruda 48/ 11, 41, 2001).

Die Vereine versuchen, neue Mitglieder anzuziehen, indem sie zweisprachig werden. Im Bericht eines Vereins über die Beitrittsfeier zur Europäischen Union konnte man in einem Artikel lesen, dass ,das gemeinsame Ziel in der Erhaltung des Slowenentums und der slowenischen Sprache in diesem Teil Europas liegt “6 (Gorjup Posinković 2004: 21) und ein paar Zeilen später, dass ,das Kulturprogramm ganz auf die jungen Generationen abgestimmt war [...], die ganze Moderation verlief zweisprachig. Besonders Letzteres zeigt, dass sich die slowenischen Vereine auch dem deutschen Raum öffnen und so ihre Lebendigkeit und Frische und den Sinn ihres Wirkens erhalten wollen"“7 (ibd., 22).

Beim Besuch slowenischer Veranstaltungen konnte ich oft beobachten, dass junge Leute, auch wenn sie Slowenisch können, sich untereinander auf Deutsch unterhalten. Dies erklären sie damit, dass sie die Dialekte der anderen Sprecher nicht verstünden; die Slowenischkenntnisse sind nämlich bei der zweiten Generation auf Dialekte beschränkt.

\subsubsection{SLOWENISCHUNTERRICHT}

Die Anfänge des Slowenischunterrichts gehen auf das Jahr 1970 zurück, als „die ersten jugoslawischen Schulen oder, besser gesagt, besonderen Klassen [mit] zusätzlichem Unterricht für Kinder jugoslawischer Arbeiter ${ }^{\text {“8 } 8}$ eröffnet wurden (Prešeren 1971: 22). Wegen der niedrigen Anzahl slowenischer Kinder wurde diesen aber oft der Unterricht in serbokroatischer Sprache aufgezwungen. ${ }^{9}$

In den 1970er und 1980er Jahren war die Zahl der Kinder hoch, später ist sie zurückgegangen. Die Gründe dafür waren die wirtschaftlichen Krisen in Deutschland und Jugoslawien, deretwegen die jugoslawischen Arbeiter nicht nach Jugoslawien zurückkehrten. Der Slowenischunterricht war nämlich vorerst mit der zu erwartenden Rückkehr nach Slowenien verbunden. Als diese immer unwahrscheinlicher wur-

5 „Veliko klubov je propadlo zaradi finančnih problemov in sektaštva, ker si demokracijo razlaga vsak po svoje. Veliko nezadovoljstva povzroča tudi meddruštveno nagajanje, nevoščljivost, politične spletke, zaradi česar prihaja do bojkotiranja prireditev ali do organiziranja privlačnejših konkurenčnih prireditev ob istem času."“

6 ,[...] skupni cilj ohranjati slovenstvo in slovenski jezik v tem delu Evrope“.

7, ,.... kulturni program $\mathrm{v}$ celoti uglašen na mlade generacije [...], povezovanje programa pa je v celoti potekalo dvojezično. Prav slednje najzgovorneje priča o tem, da se želijo slovenska društva odpirati tudi v nemški prostor in $\mathrm{s}$ tem ohranjati svojo živahnost, svežino in smisel delovanja“.

8 ,[...] prve jugoslovanske šole ali, bolje rečeno, posebni razredi, dopolnilni pouk za otroke jugoslovanskih delavcev“.

9 „S: Bist du in die slowenische Schule gegangen? Marjan, 2. Gen., geb. 1970: Nein. In die slowenische Schule deshalb, weil ... in (...) gab es nur eine jugoslawische Schule ... dadurch wurde dort nur Serbokroatisch gelernt." (S: Si hodil v slovensko šolo? Marjan: Ne. [Fa] slovensko šolo zato, ker ... [Va] (...) je bla samo jugoslovanska šola ... s tem, da so potem tam samo učili srbohrvaščino.) 
de, begann der Slowenischunterricht in seiner damaligen Konzeption seinen Sinn zu verlieren:

„Seien wir uns dessen bewusst, dass dem Zusatzunterricht und überhaupt allen Formen des getrennten Schulwesens für ausländische Kinder in der BRD die letzte Stunde schlägt. Offizielle Kreise in Bonn kündigen das klar mit der These an, dass sie sich kein ,ausländisches Ghetto' leisten können, wobei sie dabei vor allen an die Armee türkischer Kinder auf den Straßen denken, dabei aber genauso und noch mehr auf die Kinder jener kleinen Nationen zielen, die wegen der Isolierung von den Landsleuten noch viel mehr der Assimilation ausgesetzt sind. Bonn greift krampfhaft das so genannte ,bayerische Modell‘ an, das den ausländischen Kindern den größten Teil der Schulung in ihrer Muttersprache ermöglicht und das auf der (konservativen) politischen Strategie beruht, nach der die ausländische Arbeiterschaft immer an der Schwelle gehalten werden soll. Mit ihren schönen Worten gesagt: den Ausländern soll man immer die Tür für die Rückkehr offen halten“"10 (Vrata se zdaj zapirajo z druge strani [Die Tür schließt jetzt von der anderen Seite], Artikel aus der Zeitung Delo; Rodna gruda XXVII/2, 13, 1980).

Heute sind durch die geringe Kinderzahl weniger Lehrer als in der Vergangenheit angestellt, der Unterricht ist oft so organisiert, dass in einer Klasse Kinder unterschiedlichen Alters und mit unterschiedlichen Sprachkenntnissen sitzen. Die Arbeit ist wegen dieser großen Unterschiede in den Klassen und durch den Unterricht an unterschiedlichen Orten - die Lehrer fahren jede Woche mehrere hundert Kilometer - schwer, es wird aber mit viel Hingabe gearbeitet, so dass unter den gegebenen Umständen die Resultate auch gut sind. ${ }^{11}$

10 „Zavedajmo se, da dopolnilnemu in nasploh vsem oblikam ločenega šolstva za tuje otroke v ZRN bije ura. Uradni bonski krogi to že jasno napovedujejo s tezo, da si ne morejo več privoščiti 'tujskega geta', misleč sicer prvenstveno na armado turških otrok na ulicah, merijo pa ravno tako in še huje na otroke tistih malih nacij, ki so zaradi izoliranosti od rojakov še mnogo bolj izpostavljeni asimilaciji. Bonn krčevito napada tako imenovani 'bavarski model', ki omogoča tujim otrokom pretežni del šolanja v materinščini in ki temelji na (konservativni) politični strategiji, po kateri je treba držati tujsko delavstvo vedno na pragu. Z njihovimi lepimi besedami povedano: tujcem je treba držati odprta vrata za vrnitev v domovino."

11 „Slowenisch besuchen sie ... [in Ulm, Anm. S. Š.] einmal alle vierzehn Tage ... sie haben nur Unterricht.“ Die Arbeit der Lehrer ist schwer, „,weil plötzlich die Idee aufkam, dass alle in einer Gruppe arbeiten müssen. Das ist unmöglich ... Wissen Sie, ein sechsjähriges Kind und ein vierzehnjähriges ... in einer Gruppe. Das geht nicht, aber natürlich ... so helfen sich die Slowenen eben, weil es zu wenig ... Jugend gibt"' (Primož, 1. Gen., 1942/ 1976). (Slovenščino obiskujejo ... [in Ulm, Anm. S. Š.] enkrat na štirnajst dni ... majo samo pouk. ... ker je kar naenkrat prišla ideja, da morajo v eni skupini delat. To je nemogo$\check{c}$ e ... Veste, šestleten otrok pa štirnajstletni ... visti skupini. Ne gre. Ampak sevede ... to si Slovenci pač pomagajo, ker je premal ... mladine). 


\subsubsection{KIRCHE}

In Baden-Württemberg sind seit 1960 slowenische Priester tätig. Slowenische Pfarreien sorgen für die Organisation von Messen in slowenischer Sprache, für slowenische Zeitungen und Zeitschriften, für die Bibliothek (in Stuttgart gibt es 2000 Bücher), sie organisieren unterschiedliche Veranstaltungen (Filmabende, Ausflüge, Pilgerfahrten, Pfingsttreffen, Familienseminare ...), sorgen für Kontakte mit Slowenen innerhalb oder außerhalb Sloweniens und arbeiten mit der in Deutschland aufgewachsenen slowenischen Jugend.

1970 hat die slowenische Pfarre in Stuttgart auch Unterricht in slowenischer Sprache organisiert, die so genannte Samstagsschule. Dort haben die Kinder Religionsunterricht gehabt und Slowenisch gelernt. Die Priester haben die Eltern ermahnt, ,ihre Kinder die Sprache zu lehren, damit sich die Kinder nach der Rückkehr nicht wie Fremde fühlen werden. Auch wenn die Kinder in Deutschland bleiben, wäre es nützlich, die slowenische Sprache zu können. In Slowenien haben sie viele Verwandte, die sie besuchen und mit denen sie Briefwechsel führen werden “"12 (Med nami povedano [Unter uns gesagt], zitiert nach Šket - Turk 2000). Dieses Zitat zeigt, dass schon zehn Jahre vor der Zeitung Delo (vgl. Anm. 10) darauf aufmerksam gemacht wurde, dass der Sinn des Slowenischunterrichts nicht nur an eine mögliche Rückkehr gebunden und dass es wichtig ist, Slowenisch zu lernen, auch wenn die Eltern wissen, dass die Kinder wahrscheinlich in Deutschland bleiben werden.

\subsubsection{UNIVERSITÄT}

Slowenisch wird in Deutschland nicht als Einzelfach studiert, sondern im Rahmen der Lektorate an den Instituten für Slawistik angeboten. Slowenische Lektorate bieten den Slowenen die Möglichkeit, ihre Sprachkenntnisse zu verbessern. Ich habe leider keine Daten über andere Lektorate, für Tübingen kann ich aber feststellen, dass von 1999 bis 2004, als ich dort als Lektorin gearbeitet habe, nur fünf Slowenen ${ }^{13}$ das Lektorat besucht haben.

12, ,...] da naučijo svoje otroke jezika, da se otroci ob povratku v domovino ne bodo počutili kot tujci. Tudi če bi otroci ostali v Nemčiji, bi bilo koristno, da znajo slovenski jezik. V Sloveniji imajo veliko sorodnikov, ki jih bodo obiskovali in se z njimi dopisovali.““

13 Nach Angaben des slowenischen Informationszentrums in Stuttgart sollen in Baden-Württemberg 30.000 Slowenen leben (http://www.triglav-stuttgart.si/default.asp?stran=projekt, 10. 2. 2008). 


\section{Literatur}

Drnovšek 1996: $\quad$ Marjan Drnovšek, „Ti si naša misel, domovina ...“, in: Slovenska kronika XX. stoletja 1941-1995, Ljubljana, 363

Fishman 1966:

Joshua A. Fishman, Language Maintenance and Language Shift as a Field of Inquiry (A definition of the field and suggestions for its further development), in: Language Loyalty in the United States: The Maintenance and Perpetuation of Non-English Mother Tongues by American Ethnic and Religious Groups, London

Gorjup Posinković 2004: Vida Gorjup Posinković, V Evropo povezani, in: Slovenija svet I/2, 20-22

Horvat - Lavš 1995: Martin Horvat, Katarina Lavš, Iz spomina v spomin. Ob 25-letnici slovenske župnije v Berlinu: 1969-1994, Berlin

Krevs 1992:

Uršula Krevs, Govorno vedenje pri Slovencih v Nemčiji, Ljubljana

Prešeren 1971:

Jože Prešeren, Prve jugoslovanske šole v Nemčiji, in: Rodna gruda XVIII/1, 22

Skutnabb-Kangas 1981: Tove Skutnabb-Kangas, Bilingualism or Not: The Education of Minorities, Clevedon and Philadelphia

Skutnabb-Kangas 1991: Tove Skutnabb-Kangas, Bilingvizam da ili ne, Beograd

Slavec 1982:

Stölting 1980: $\quad \begin{aligned} & \text { Wilfried Stölting, Die Zweisprachigkeit jugoslawischer Schüler in der } \\ & \text { Bundesrepublik Deutschland (= Balkanologische Veröffentlichungen }\end{aligned}$ Ingrid Slavec, Slovenci v Mannheimu, Ljubljana Bundesrepublik Deutschland (= Balkanologische Veröffentlichungen 3), Wiesbaden

Šket - Turk 2000: $\quad$ Janez Šket, Ciril Turk, Slovenci na Württemberškem - nova domovi-

Štumberger 2005: $\quad$ Saška Štumberger, Slovenski selitveni tokovi v Nemčijo in spreminjana v Cerkvi, Ljubljana

Štumberger 2006a: nje vloge slovenščine, in: Dve domovini 22, 95-114 $\begin{array}{ll}\text { Štumberger 2006b: } & \text { Slavistična revija 54/1, 41-60 } \\ \text { Saška Stumberger, Slovenščina od zunaj kot drugi/tuji jezik Sloven- }\end{array}$ Saška Stumberger, Dvojezičnost na primeru Slovencev v Nemčiji, in:

cev: slovenske izseljenske skupnosti v Evropski uniji s poudarkom na opisu položaja v Nemčiji, in: Zvon 9/3, 46-49

Štumberger 2007: $\quad$ Saška Štumberger, Slovenščina pri Slovencih v Nemčiji, Ljubljana

A b s t r a c t: The Slovenian Language and Bilingualism among Slovenians in Germany. This article is based on a study conducted during the author's five-year stay in Germany from 1999 to 2004 among bilingual adult Slovenians living and working in that country. The aim of the study was to analyse where the Slovenian language is used today among these bilingual adult speakers (Slovenian clubs and associations, churches and universities; it also looked at the possibility to attend Slovenian classes as an extra-curricular activity). All the results presented in the study come from data either derived from interviews and questionnaires or obtained by methods of participatory observation.

K e y w or d s : bilingualism, Slovenians in Germany, socio-linguistics, Slovenia

Saška Štumberger

FF, Oddelek za slovenistiko

Aškerčeva 2

1000 Ljubljana, Slowenien

saska.stumberger@guest.arnes.si 